\title{
Avaliação da assistência puerperal no âmbito da atenção básica
}

\author{
Assessment of puerperal assistance within the basis of basic care
}

Evaluacíon de la asistencia puerperal dentro de la base de cuidado básico

Mikellayne Barbosa Honorato ${ }^{1 *}$, Rayanne Lúcia de Oliveira Campos ${ }^{1}$, Maisa Gonçalves de Araújo, Érika Maria Alves da Silva ${ }^{1}$, Priscila Santos Alves Melo르. Danielle Santos Alves ${ }^{1}$

\section{RESUMO}

Objetivo: Avaliar a assistência puerperal no âmbito da rede de atenção básica de saúde. Métodos: Trata-se de um estudo transversal, desenvolvido em nove Unidades Básicas de Saúde (UBS), em um munícipio do PE. Para o cálculo da amostra, foi utilizada uma amostragem estratificada e adotada a prevalência de $50 \%$, admitindo o estudo de populações finitas. A amostra final foi de 156 prontuários. Resultados: As puérperas apresentaram idade entre 15 a 35 anos (84,1\%), estado civil solteira (39,2\%) e a maioria concluiu o ensino médio (26,3\%). Dos 156 prontuários avaliados, em 114 havia consulta de puerpério documentada, com cobertura de $73,1 \%$, sendo a maioria (55,8\%) realizada por enfermeiros. Houve uma elevada predominância de ausência de registros de sinais vitais e condutas realizadas. Conclusão: Observou-se que grande parte das mulheres teve acesso a consulta puerperal, porém, atenta-se para os registros precários dos prontuários. Faz-se necessário a realização de mais pesquisas, para uma melhor compreensão do cenário abordado e identificação das causas das falhas de registros.

Palavras-chave: Período pós-parto, Atenção primária à saúde, Saúde materna.

\begin{abstract}
Objective: To evaluate puerperal care within the primary health care network. Methods: This is a cross-sectional study, developed in nine Basic Health Units (UBS), in a municipality in the PE. For the calculation of the sample, a stratified sampling was used and the prevalence of $50 \%$ was adopted, admitting the study of finite populations. The final sample was 156 records. Results: As puerperal women age between 15 to 35 years (84.1\%), single marital status (39.2\%) and the majority defined high school (26.3\%). Of the 156 medical records acquired, 114 had documented postpartum consultation, covering $73.1 \%$, with the majority $(55.8 \%)$ being performed by nurses. There was a high prevalence of records of vital signs and conducts performed. Conclusion: It was observed that most women had access to puerperal consultation, however, attention was paid to the poor records of the medical records. It is necessary to carry out more research to better understand the scenario addressed and identify the causes of record failures.
\end{abstract}

Keywords: Postpartum period, Primary health care, Maternal health.

\section{RESUMEN}

Objetivo: Evaluar la atención puerperal dentro de la red de atención primaria de salud. Métodos: Se trata de un estudio transversal, desarrollado en nueve Unidades Básicas de Salud (UBS), en un municipio del PE. Para el cálculo de la muestra se utilizó un muestreo estratificado y se adoptó la prevalencia del $50 \%$, admitiéndose el estudio de poblaciones finitas. La muestra final fue de 156 registros. Resultados: Como edad posparto, edad entre 15 a 35 años $(84,1 \%)$, estado civil soltero $(39,2 \%)$ y bachillerato más definido $(26,3 \%)$. De las 156 historias clínicas adquiridas, 114 tenían consulta posparto documentada, lo que cubre el 73,1\%, siendo la mayoría $(55,8 \%)$ realizada por enfermeras. Hubo una alta prevalencia de registros de constantes vitales y conductas realizadas. Conclusión: Se observó que la mayoría de las mujeres tuvo acceso a la consulta puerperal, sin embargo, se prestó atención a los malos antecedentes de las historias clínicas. Es necesario realizar más investigaciones para comprender mejor el escenario abordado e identificar las causas de las fallas en los registros.

Palabras clave: Periodo posparto, Atención primaria de salud, Salud materna.

${ }^{1}$ Universidade Federal de Pernambuco, Recife - PE. *E-mail: mikellaynebhonorato@gmail.com 


\section{INTRODUÇÃO}

O puerpério, também denominado pós-parto, é uma fase do ciclo grávido-puerperal que, do ponto de vista biológico, se inicia após a dequitação da placenta e termina com o retorno do organismo materno às condições passíveis de involução, por volta de seis semanas após o parto. Essa fase é um momento crítico e de transição na vida da mulher e da família, também marcada por modificações biológicas, psicológicas, comportamentais e socioculturais (CORTEZ PM e VALIM CGC, 2014; SOUSA FET, et al., 2015) que envolve, desde sentimentos gerados pela necessidade de ajuste ao filho real, às transformações corporais e à mudança na configuração familiar, exigindo esforço psíquico da mulher (CORRÊA MSM, et al., 2017).

Segundo o Ministério da Saúde (MS), a consulta puerperal é fundamental para saúde da mãe e da criança, devendo ser realizada ainda nas primeiras semanas pós-parto. Cabe aos profissionais de saúde, durante as consultas de pré-natal, incentivar e reforçar a importância do retorno da mulher e recém-nascido aos serviços de saúde, além de agendar a visita domiciliar.

Logo, a assistência pré-natal, ao parto e ao puerpério deve ser pensada também como uma oportunidade para que o sistema atue integralmente na promoção e, eventualmente, na recuperação desse público-alvo. Além disso, é um momento oportuno para realizar, por exemplo, a prevenção do câncer cérvico uterino, investigar possíveis complicações físicas ou psíquicas (BRASIL, 2018). Durante a consulta de puerpério deve haver uma sistematização da assistência, buscando englobar todos os aspectos pertinentes àquele momento. Deve haver um acolhimento com escuta qualificada e uma avaliação global, abordando o cartão gestacional, os dados do RN, dados do parto e queixas. Além disso, é imprescindível a realização do exame físico geral e específico e a elaboração de um plano de conduta, baseado nos achados (CUNHA MR, et al, 2018; SILVA LP, et al., 2020).

Uma pesquisa realiza mostra que a visita/consulta puerperal no âmbito da atenção básica ainda apresenta falhas e geram sentimentos de insatisfação nas puérperas, além de queixas como consulta assistemática e descontinuada, acesso difícil ao médico, atenção focada no bebê e escassez de exame físico e anamnese (CORRÊA MSM, et al., 2017).

No âmbito da atenção primária a saúde, a visita domiciliar no pós-parto é um momento ideal e oportuno para identificação precoce de agravos a saúde materna e do recém-nascido. Nesta ocasião deve se empregar um olhar holístico sobre o binômio mãe-filho, assim como para seu núcleo familiar, objetivando detectar sinais que possam evoluir para agravos importantes, como a depressão pós-parto, dificuldades na amamentação e alterações de crescimento ou físicas no recém-nascido, sendo idealmente a primeira consulta até $\circ 7^{\circ}$ dia pós-parto e a segunda entre o 30 e 40 dias pós-parto (BARATIERI T e NATAL S, 2019; SBIBAE, 2019).

É necessário que os profissionais envolvidos em qualquer instância do processo assistencial entendam a importância de sua atuação e da necessidade de avaliar o conhecimento técnico específico e o compromisso por resultados satisfatórios, levando em consideração o significado destes resultados para cada mulher e para os índices de saúde do país (BRASIL, 2018; ALVARENGA SC, et al., 2017). Os profissionais de saúde, por vezes, tratarem o ciclo gravídico-puerperal de forma isolada do contexto gravídico-puerperal. Por esse motivo, este período tem sua assistência fragmentada.

Considerando estes aspectos, com a relação à importância da consulta puerperal para continuação do cuidado, detecção de agravantes e educação em saúde da mulher nessa nova fase da vida, o estudo em questão teve como objetivo avaliar a assistência puerperal prestada a mulheres assistidas na rede básica de saúde.

\section{MÉTODOS}

Trata-se de um estudo transversal, exploratório, quantitativo, realizado no período de março a maio de 2018, em nove Unidades Básicas de Saúde (UBS), em um munícipio do PE. Teve como objetivo investigar os registros dos prontuários de puérperas atendidas pela rede de atenção básica de saúde quanto à qualidade da assistência prestada. Para o cálculo da amostra, foi utilizada uma amostragem estratificada e adotada a prevalência de $50 \%$, admitindo o estudo de populações finitas. A amostra foi constituída inicialmente por 163 mulheres e seus respectivos prontuários, que realizaram as consultas de pré-natal e puerpério nas UBS selecionadas, já incluídos os percentuais de eventuais perdas. Contudo, existiram alguns obstáculos para a obtenção da amostra final, sendo está de 156 prontuários. 
No que diz respeito às dificuldades de acesso aos prontuários, atuaram como responsáveis: a transição de algumas unidades para prontuário eletrônico, dificultando a coleta de dados pela restrição de acesso ao sistema; a indisponibilidade dos prontuários; a baixa colaboração de alguns profissionais de saúde; e a ausência de registros no prontuário. Os motivos citados inviabilizaram o alcance da amostra total, caracterizando-se como um viés da pesquisa. Foram incluídos os prontuários das mulheres atendidas entre outubro de 2017 e março de 2018 e excluídos os prontuários incompletos, ou seja, em que as mulheres iniciaram o seu pré-natal e mudaram de endereço ou abandonaram a assistência.

O instrumento utilizado para realizar a coleta de dados conteve perguntas relacionadas aos dados de identificação, sociodemográficos, história da gestação e parto, dados relacionados às consultas do puerpério e possíveis orientações. Este instrumento elaborado e transcrito para um formulário eletrônico no Google Forms, para facilitar a coleta nas UBS.

A pesquisa é derivada de um projeto maior intitulado "Adequação do pré-natal em unidades básicas de saúde baseada nos critérios do ministério da saúde e organização mundial da saúde" e foi aprovada pelo Comitê de Ética em Pesquisa do Centro de Ciências da Saúde da Universidade Federal de Pernambuco, certificado pelo CAE 81235717.5.0000.5208 e cumpre todas as exigências estabelecidas pela Resolução n466/12, do Conselho Nacional de Saúde.

\section{RESULTADOS}

Dos 156 prontuários disponibilizados pelas nove unidades de saúde da família (USF) participantes do estudo, apenas 114 prontuários possuíam consulta de puerpério documentada, ou seja, a cobertura da assistência puerperal foi de $73,1 \%$.

Com relação aos dados sociodemográficos, observou-se a predominância de puérperas com idades entre 15 a 35 anos de idade $(84,1 \%)$, sendo a idade média de 29 anos, solteiras $(39,2 \%)$ e que se autodeclararam negras/pardas (37,2\%). Nota-se que as informações acerca do nível de escolaridade foram negligenciadas pelos profissionais responsáveis pela consulta, visto que em mais da metade dos prontuários $(51,9 \%)$ este dado não estava registrado. Dentre os registros, houve a prevalência de mulheres com o ensino médio $26,3 \%$ (Tabela 1).

Tabela 1 - Caracterização sociodemográfica das puérperas das nove USF's, 2018.

\begin{tabular}{|c|c|c|}
\hline Variável & $\mathrm{N}=156$ & $100 \%$ \\
\hline \multicolumn{3}{|l|}{ Idade } \\
\hline$<15$ anos & 7 & 4,5 \\
\hline 1535 anos & 132 & 84,1 \\
\hline$>35$ anos & 13 & 8,3 \\
\hline Não registrado & 4 & \\
\hline Idade mínima & \multicolumn{2}{|c|}{13 anos } \\
\hline Idade máxima & \multicolumn{2}{|c|}{42 anos } \\
\hline Idade média (desvio padrão) & \multicolumn{2}{|c|}{29 anos $(+-6,76)$} \\
\hline \multicolumn{3}{|l|}{ Raça / Cor (autodeclarado) } \\
\hline Amarela & 5 & 3,2 \\
\hline Branca & 14 & 9,0 \\
\hline Negra/preta/parda & 58 & 37,2 \\
\hline Não registrado & 79 & 50,6 \\
\hline \multicolumn{3}{|l|}{ Estado Civil } \\
\hline Casada & 27 & 17,3 \\
\hline Solteira & 40 & 25,6 \\
\hline União estável & 35 & 22,4 \\
\hline Não registrado & 54 & 34,6 \\
\hline \multicolumn{3}{|l|}{ Escolaridade } \\
\hline Ensino fundamental & 27 & 17,3 \\
\hline Ensino médio & 41 & 26,3 \\
\hline Ensino superior & 7 & 4,5 \\
\hline Não registrado & 81 & 51,9 \\
\hline
\end{tabular}

Fonte: Honorato MB, et al., 2020. 
Quanto aos dados referentes ao parto das puérperas, chama-se atenção para o alto ausência de registro deste dado nos prontuários $(70,2 \%)$. Naqueles onde este dado estava presente, observa-se que metade dos partos ocorreram em maternidades de alto risco e metade nas de baixo risco, com 13 partos cada, tendo o parto vaginal a prevalência de $42,9 \%$.

Com relação aos dados do recém-nascido os dados referentes ao apgar no primeiro e quinto minuto, estava presente em apenas $45,6 \%$. Apesar disto, observa-se que os recém-nascidos tiveram prevalência de score entre 9 e 10 no primeiro e quinto minuto, respectivamente $(35,1 \%$ e $43 \%)$. Com relação ao peso no nascimento, houve a predominância de recém-nascidos com peso entre $2500 \mathrm{~g}$ a $4500 \mathrm{~g}(55,3 \%)$, com também alta prevalência de não registrados $(41,2 \%)$ (Tabela 2).

Tabela 2 - Dados referentes ao parto das puérperas das 9 USF's, 2018.

\begin{tabular}{|c|c|c|}
\hline cv-. & $\mathrm{N}=114$ & $100 \%$ \\
\hline \multicolumn{3}{|l|}{ Local de parto } \\
\hline Maternidade de Alto Risco & 13 & 11,4 \\
\hline Maternidade de Risco Habitual & 13 & 11,4 \\
\hline Entidade filantrópica & 4 & 3,5 \\
\hline Maternidade de rede privada & 3 & 2,6 \\
\hline Ambulância & 1 & 0,9 \\
\hline Não registrado & 80 & 70,2 \\
\hline \multicolumn{3}{|l|}{ Tipo De parto } \\
\hline Cesárea & 22 & 19,3 \\
\hline Vaginal & 49 & 42,9 \\
\hline Não registrado & 43 & 37,7 \\
\hline \multicolumn{3}{|l|}{ Apgar no primeiro minuto } \\
\hline 9 a 10 & 40 & 35,1 \\
\hline 7 a 8 & 10 & 8,8 \\
\hline$<7$ & 2 & 1,7 \\
\hline Não registrado & 62 & 54,4 \\
\hline \multicolumn{3}{|l|}{ Apgar no quinto minuto } \\
\hline 9 a 10 & 49 & 43 \\
\hline 7 a 8 & 3 & 2,6 \\
\hline$<7$ & 0 & 0 \\
\hline Não registrado & 62 & 54,4 \\
\hline \multicolumn{3}{|l|}{ Peso ao nascer } \\
\hline$<2500 \mathrm{~g}$ & 4 & 3,5 \\
\hline De $2500 \mathrm{~g}$ a 4500 & 63 & 55,3 \\
\hline$>4500 \mathrm{~g}$ & 0 & 0 \\
\hline Não registrado & 47 & 41,2 \\
\hline
\end{tabular}

Fonte: Honorato MB, et al., 2020.

No que diz respeito às questões abordadas na consulta de puerpério, viu-se que apenas $18,4 \%$ dos prontuários tinham registro dos sinais vitais, a verificação dos lóquios foi registrada em somente $25,4 \%$, a avaliação da involução uterina teve prevalência de $8,8 \%$.

Com relação à prescrição de fármacos no puerpério, o sulfato ferroso foi constatado em apenas 26 prontuários, dentre os 114 observados, não estando claro se não foi prescrito ou não foi registrado. Observouse ainda, a ocorrência de uma prescrição de Metronidazol e uma de Lanolina (Tabela 3). 
Tabela 3 - Dados sobre a consulta puerperal registrados nos prontuários das mulheres assistidas nas 09 UBS's, 2018.

\begin{tabular}{|c|c|c|}
\hline Variável & $\mathrm{N}=114$ & $100 \%$ \\
\hline \multicolumn{3}{|l|}{ Teve acesso à consulta puerperal } \\
\hline Não & 42 & 26,9 \\
\hline Sim & 114 & 73,1 \\
\hline \multicolumn{3}{|l|}{ Avaliação de Sinais vitais } \\
\hline Sim & 21 & 18,4 \\
\hline Não registrado & 93 & 81,6 \\
\hline \multicolumn{3}{|l|}{ Avaliação dos Lóquios } \\
\hline Sim & 29 & 25,4 \\
\hline Não registrado & 85 & 74,6 \\
\hline \multicolumn{3}{|l|}{ Avaliação da involução uterina } \\
\hline Sim & 10 & 8,8 \\
\hline Não registrado & 104 & 91,2 \\
\hline \multicolumn{3}{|l|}{ Foi prescrito medicações } \\
\hline Sim & 28 & 24,6 \\
\hline Não registrado & 86 & 75,4 \\
\hline \multicolumn{3}{|l|}{ Medicamentos prescritos } \\
\hline Metronidazol & 1 & 0,9 \\
\hline Lanolina & 1 & 0,9 \\
\hline Sulfato Ferroso & 26 & 22,8 \\
\hline Não registrado & 86 & 75,4 \\
\hline \multicolumn{3}{|l|}{ Qual o profissional que realizou a consulta } \\
\hline Enfermeiro(a) & 60 & 52,6 \\
\hline Médico(a) & 4 & 3,5 \\
\hline Não registrado & 50 & 43,8 \\
\hline \multicolumn{3}{|l|}{ Condutas e orientações registradas } \\
\hline Orientação sobre amamentação & 8 & 7,0 \\
\hline Orientação sobre cuidados gerais com o recém-nascido & 19 & 16,6 \\
\hline Solicitação de exames & 2 & 1,7 \\
\hline Manter sulfato ferroso & 1 & 0,9 \\
\hline Encaminhamento ao planejamento familiar & 3 & 2,6 \\
\hline Orientações gerais sobre o pós-parto & 6 & 5,3 \\
\hline Sem registro de orientações & 75 & 65,8 \\
\hline
\end{tabular}

Fonte: Honorato MB, et al., 2020.

Constatou-se que as consultas de puerpério foram realizadas, em sua maioria, por enfermeiros $(52,6 \%)$, mesmo não sendo possível de identificar quem a realizou em $43,8 \%$. Apenas $34,7 \%$ das mulheres receberam algum tipo de orientação sobre o período pós-parto, sendo prevalentes orientações sobre o recém-nascido. Em $65,8 \%$ dos prontuários, não foi identificado o registro destas orientações.

\section{DISCUSSÃO}

Em relação à análise dos dados obstétricos e qualidade da assistência puerperal, percebe-se que 42 mulheres não compareceram à consulta de retorno do puerpério. Tal fato reflete falha na assistência, uma vez que a Organização Mundial de Saúde (OMS) e o Ministério da Saúde idealizam um cuidado contínuo e de qualidade durante o ciclo gravídico puerperal (BRASIL, 2018). Uma vez constatada falha na adesão das mulheres a comparecerem a consulta puerperal, deve-se fazer a busca ativa e/ou visita domiciliar, a fim de prevenir situações de risco materno e neonatal comuns na primeira semana após o parto (VILELA MLF e PEREIRA QLC, 2018).

Além disso, o vínculo formado entre o profissional e a mulher ao longo do pré-natal, assim como as orientações realizadas nesse período, possibilita maior adesão às consultas no puerpério (SILVA EC, 2017). Outra estratégia para aumentar o comparecimento à consulta puerperal é potencializar as recomendações quanto a importância desse acompanhamento durante a alta hospitalar, buscando garantir a continuidade de cuidados (FUSQUINE RS, et al., 2019). 
O acompanhamento pós-parto de qualidade deve envolver as principais demandas inerentes a esse período, nas esferas biológicas, sociais e emocionais. Sendo assim, a anamnese, a aferição de sinais vitais, realização de exame físico, avaliação ginecológica e do estado psíquico, bem como orientações acerca da amamentação, sono e repouso, atividades sexuais, planejamento familiar, uso de medicamentos e cuidados com o recém-nascido, devem compor a consulta de pós-parto (PEREIRA MC, et al., 2014; MERCADO NC, et al., 2017).

Identificou-se que os resultados referentes à faixa etária das mulheres participantes do estudo (entre $15 \mathrm{e}$ 35 anos) assemelham-se a outras pesquisas em contexto hospitalar (OMS, 2016; THULER ACMC, 2016). Essa informação é relevante no que se refere à antecipação de complicações próprias de cada período etário, viabilizando uma assistência preventiva.

Considerando ainda que essas mulheres estão no período fértil (faixa etária entre 10 a 49 anos), é imprescindível que estas tenham acesso ao planejamento familiar (RODRIGUES AP, 2016). Priorizando informações como o intervalo interpartal para concepção e orientação sobre a escolha do método anticoncepção mais adequado para a puérpera, garantindo o direito a escolha livre e consciente dessas mulheres (MONTENEGRO CAB e REZENDE FILHO J, 2013; BRASIL, 2010).

Em relação ao estado civil, algumas mulheres se declararam solteiras. Esse dado pode estar associado a baixa adesão às consultas de pré-natal e puerpério, assim como a terapêutica indicada, pois, a ausência de um parceiro (a), no sentido de rede de apoio, principalmente para aquelas que possuem mais de um filho, contribui para tal, visto que, muitas delas, precisam ausentar de atividades diárias, de trabalho ou domésticas (SILVA JMB e NUNES MA, 2017; FUSQUINE RS, et al., 2019).

Nesta pesquisa, observou-se que a variável escolaridade estava ausente em $51,9 \%$ dos prontuários. A informação sobre a escolaridade da puérpera é de suma importância, pois contribui na identificação da capacidade cognitiva dessas mulheres e, consequentemente, colabora no sucesso das ações educativas. A alta escolaridade está relacionada a resultados positivos antes, durante e após a gestação, diferente da baixa escolaridade que pode facilitar a propagação de inverdades sobre o puerpério e cuidados com o recémnascido.

A identificação do perfil sociodemográfico dessas mulheres se mostra essencial para permitir o direcionamento do cuidado e aprofundar as intervenções clínicas levando em consideração as particularidades das puérperas e suas necessidades baseado no perfil sociodemográfico e cultural, não apenas biológico (OMS, 2016; THULER ACMC, et al., 2016; SILVA JMB e NUNES MA, 2017).

No que concerne à incidência de consultas realizadas pelos profissionais, destaca-se uma maior predominância $(55,8 \%)$ pelos profissionais de enfermagem. Resultado semelhante pode ser observado em outros estudos, onde o enfermeiro é o profissional que primeiro atende a mulher no retorno às consultas puerperais (SILVA LLB, et al, 2016; CARVALHO MJLN, et al., 2019).

Durante a consulta puerperal, devem ser analisadas as condições que elevam a morbimortalidade materna, tais como: doença tromboembólica venosa, anemia, hemorragia pós-parto, diabetes e hipertensão gestacional (RODRIGUES AP, et al., 2016; BRASIL, 2013). Desta maneira previne-se as repercussões negativas de uma morte materna, uma vez que a morte de uma mãe representa uma perda social para o recém-nascido, os outros filhos e da família como um todo (ANDRADE RD, et al., 2015).

O puerpério é uma oportunidade crucial para os profissionais de saúde darem continuidade ao cuidado, bem como reforçar e apoiar as informações a serem dadas às mulheres nesta nova etapa de suas vidas, incluindo a detecção de desvios da normalidade com relação ao biológico, até orientações acerca da sua saúde e bem-estar, tais como: mudanças no estilo de vida, aconselhamento sobre planejamento familiar, entre outros.

Tendo o profissional de saúde, com ênfase na equipe multidisciplinar da $A B$, a responsabilidade e dever em orientar essas mulheres, acompanhantes e familiares acerca dos cuidados e medidas a serem tomadas, antes, durante e após o nascimento da criança. Logo, acredita-se que o estudo contribuiu significativamente para a população e profissionais de saúde, dado que, estimula a conscientização da equipe multiprofissional ao preenchimento correto de todos os campos do prontuário e a realização das condutas assistenciais durante todo o período gestacional e puerperal, viabilizando uma assistência de excelência para esse público. 
Ademais, a pesquisa oportuniza verificar a importância do preenchimento do prontuário, visto que, tratase de documento jurídico do paciente, garantindo a ele legalmente quais foram as condutas realizadas pelos profissionais, sendo também fonte para pesquisas científicas, permitindo a continuidade dos cuidados. 0 prontuário também possui impacto com relação ao respaldo legal dos profissionais, troca de informações entre a equipe multiprofissional e outros níveis de assistência e na continuidade do atendimento.

\section{CONCLUSÃO}

Neste estudo foi observado que há uma significante cobertura da consulta puerperal no distrito IV da cidade do Recife, porém o exame físico, a verificação de sinais vitais e a oferta de informações inerentes ao período foram documentas em poucos prontuários. Esses prontuários apresentam deficiências importantes quanto ao registro correto e completo das informações referentes a assistência e aos dados das mulheres. A falta de registro nos prontuários tornou difícil avaliar a qualidade da assistência pós-parto nas unidades em estudo e levantou uma importante questão sobre a fragilidade do único documento de registro das condutas dirigidas as puérperas, podendo ser um dificultador, inclusive, da tomada de decisão por outros profissionais.

\section{REFERÊNCIAS}

1. ALVARENGA SC, et al. Fatores que influenciam o desmame precoce. Aquichan, 2017; 17(1): 93-103.

2. ANDRADE RD, et. al. Fatores relacionados à saúde da mulher no puerpério e repercussões na saúde da criança. Esc Anna Nery - Rev Enferm. 2015;19(1):181-6.

3. BRASIL. MINISTÉRIO DA SAÚDE. Protocolos da Atenção Básica: Saúde das Mulheres/Ministério da Saúde, Instituto SírioLibanês de Ensino e Pesquisa - Brasília: Ministério da Saúde, 2016.

4. BRASIL. MINISTÉRIO DA SAÚDE. Secretaria de Atenção à Saúde. Departamento de Atenção Básica. Saúde sexual e saúde reprodutiva - (Cadernos de Atenção Básica, n. 26). Brasília: Ministério da Saúde, 2010: 300.

5. BRASIL. MINISTÉRIO DA SAÚDE. Secretaria de Atenção à Saúde. Departamento de Atenção Básica. Atenção ao pré-natal de baixo risco- (Cadernos de Atenção Básica, n 32). Brasília: Ministério da Saúde, 2013: 318.

6. CARVALHO MJLN, et al. Primeira visita domiciliar puerperal: uma estratégia protetora do aleitamento materno exclusivo. Revista Paulista de Pediatria, 2018; 36(1): 66-73.

7. CORRÊA MSM, et. al. Acolhimento no cuidado à saúde da mulher no puerpério. Cad. Saúde Pública, 2017; 33(3): e00136215.

8. CORTEZ PM, VALIM CGC. Consulta Puerperal A visão do enfermeiro e da puérpera. Ciência, Cuidado e Saúde, 2014; 13(1): 35-42.

9. CUNHA MR, et al. Identificação da infecção de sítio cirúrgico pós-cesariana: consulta de enfermagem. Revista Brasileira de Enfermagem, 2018; 71: 1395-1403.

10. DANTAS SLC, et. al. Representações sociais de enfermeiros da atenção primária à saúde sobre cuidado de enfermagem no pós-parto. Cogitare Enferm, 2018; 23(3).

11. FUSQUINE RS, et. al. Adesão e rejeição à consulta puerperal por mulheres de uma unidade básica de saúde da família. Arch. Health. Sci, 2019; 26(1): 37-40.

12. MERCADO NC, et. al. Cuidados e orientações de enfermagem às puérperas no alojamento conjunto. Rev. enferm. UFPE online, 2017; 3508-3515.

13. MONTENEGRO CAB, REZENDE FILHO J. Rezende obstetrícia. 12 ed. Río de Janeiro: Guanabara Koogan, 2013.

14. MUNIZ EB, et. al. Análise do boletim de Apgar em dados do Sistema de Informação sobre Nascidos Vivos registrados em um hospital do interior do estado do Ceará, Brasil. Rev Med e saúde Brasília, 2016; 191.

15. OMS. ORGANIZAÇÃO MUNDIAL DA SAUUDE. Recomendações da OMS sobre cuidados pré-natais para uma experiência positiva na gravidez. 2016;10.

16. PEREIRA MC, GRADIM CVC. Consulta puerperal: a visão do enfermeiro e da puérpera, 2014;13(265): 35-42.

17. POLES MM, et al. Sintomas depressivos maternos no puerpério imediato: fatores associados. Acta Paulista de Enfermagem, 2018; 31(4): 351-358.

18. RODRIGUES AP, et. al. Caracterização sociodemográfica e obstétrica de puérperas internadas em alojamento conjunto no Brasil. Ciencia y Enfermería, 2016; 22: 113-123.

19. SANTOS FABS, MAZZO MHSN. Puerpério e revisão pós-parto: significados atribuídos pela puérpera, 2013;17(4): 854-8.

20. SBIBAE. SOCIEDADE BENEFICENTE ISRAELITA BRASILEIRA ALBERT EINSTEIN. Nota técnica para organização da Rede de Atenção à Saúde com foco na Atenção Primária à Saúde e na Atenção Ambulatorial Especializada - Saúde da Mulher na Gestação, Parto e Puerpério. São Paulo: Hospital Israelita Albert Einstein: Ministério da Saúde, $2019 ; 56$.

21. SILVA EC, et. al. Puerpério e assistência de enfermagem: percepção das mulheres. Rev enferm UFPE online, 2017; 282633.

22. SILVA JMB, NUNES MA. Planejamento familiar: uma base de dados. J. res.: fundam. care. Online, 2017; 9(2): 510-519.

23. SILVA LLB, et al. Cuidados prestados à mulher na visita domiciliar da "Primeira Semana de Saúde Integral". Revista Gaúcha de Enfermagem, 2016; 37(3).

24. SILVA LP, et al. Assistência puerperal e a construção de um fluxograma para consulta de enfermagem. Revista Brasileira de Saúde Materno Infantil, 2020; 20(1): 101-113.

25. SOUSA FET, et al. Avanços e desafios do aleitamento materno no Brasil: uma revisão integrativa. Revista Brasileira em promoção da Saúde, 2015; 28(3): 434-442.

26. THULER ACMC, et al. Caracterização das mulheres no ciclo gravídico-puerperal e o incentivo à amamentação precoce. Rev enferm UERJ, 2018; 26: 1-6

27. VILELA MLF, PEREIRA QLC. Consulta puerperal: orientação sobre sua importância. Journal Health NPEPS, 2018; 3(1): 228-240. 